\title{
The Role of Radionuclide Imaging in the Surgical Management of Primary Hyperparathyroidism
}

\author{
Elif Hindié ${ }^{1}$, Paolo Zanotti-Fregonara ${ }^{1}$, Antoine Tabarin ${ }^{2}$, Domenico Rubello ${ }^{3}$, Isabelle Morelec ${ }^{4}$, Tristan Wagner ${ }^{5}$, \\ Jean-François Henry ${ }^{6}$, and David Taïeb ${ }^{7}$ \\ ${ }^{I}$ Department of Nuclear Medicine, Haut-Lévêque Hospital, CHU de Bordeaux, CNRS-UMR 5287, Translational and Advanced \\ Imaging Laboratory (TRAIL), University of Bordeaux, Bordeaux, France; ${ }^{2}$ Department of Endocrinology, CHU de Bordeaux, \\ University of Bordeaux, Bordeaux, France; ${ }^{3}$ Department of Nuclear Medicine, Santa Maria della Misericordia Hospital, Rovigo, \\ Italy; ${ }^{4}$ Department of Nuclear Medicine, Lyon Sud Hospital, Hospices Civiles de Lyon, Lyon, France; ${ }^{5}$ Department of Endocrine \\ Surgery, CHU de Bordeaux, University of Bordeaux, Bordeaux, France; ${ }^{6}$ Department of Endocrine Surgery, La Timone Hospital, Aix- \\ Marseille University, Marseille, France; and ${ }^{7}$ Department of Nuclear Medicine, La Timone Hospital, CERIMED, Aix-Marseille \\ University, Marseille, France
}

Learning Objectives: On successful completion of this activity, participants should be able should be able to describe (1) the role of parathyroid scintigraphy before bilateral cervicotomy or minimally invasive surgery for primary hyperparathyroidism; (2) the performance of different imaging protocols to detect multiglandular disease; (3) the appropriate imaging strategy before reoperation for persistent or recurrent hyperparathyroidism; and (4) the potential use of novel PET tracers.

Financial Disclosure: The authors of this article have indicated no relevant relationships that could be perceived as a real or apparent conflict of interest.

CME Credit: SNMMI is accredited by the Accreditation Council for Continuing Medical Education (ACCME) to sponsor continuing education for physicians. SNMMI designates each JNM continuing education article for a maximum of 2.0 AMA PRA Category 1 Credits. Physicians should claim only credit commensurate with the extent of their participation in the activity. For CE credit, SAM, and other credit types, participants can access this activity through the SNMMI website (http://www.snmmilearningcenter.org) through May 2018.

Primary hyperparathyroidism is a frequent and potentially debilitating endocrine disorder for which surgery is the only curative treatment. The modalities of parathyroid surgery have changed over the last 2 decades, as conventional bilateral neck exploration is no longer the only surgical approach. Parathyroid scintigraphy plays a major role in defining the surgical strategy, given its ability to orient a targeted (focused) parathyroidectomy and to recognize ectopic locations or multiglandular disease. This review, which represents a collaborative effort between nuclear physicians, endocrinologists, and endocrine surgeons, emphasizes the importance of performing imaging before any surgery for primary hyperparathyroidism, even in the case of conventional bilateral neck exploration. We discuss the advantages and drawbacks of targeted parathyroidectomy and the performance of various scintigraphic protocols to guide limited surgery. We also discuss the optimal strategy to localize the offending gland before reoperation for persistent or recurrent hyperparathyroidism. Finally, we describe the potential applications of novel PET tracers, with special emphasis on ${ }^{18} \mathrm{~F}$-fluorocholine.

Key Words: hyperparathyroidism; MIBI; dual-tracer; parathyroid subtraction imaging; SPECT/CT; PET/CT; ${ }^{18} \mathrm{~F}$-fluorocholine; ${ }^{11} \mathrm{C}$ methionine

J Nucl Med 2015; 56:737-744

DOI: 10.2967/jnumed.115.156018

Received Feb. 13, 2015; revision accepted Mar. 25, 2015.

For correspondence or reprints contact: Elif Hindié, Department of Nuclear Medicine, Haut-Lévêque Hospital, Avenue Magellan, 33604 Pessac, France. E-mail: elif.hindie@chu-bordeaux.fr

Published online Apr. 9, 2015.

COPYRIGHT (C 2015 by the Society of Nuclear Medicine and Molecular Imaging, Inc.
$\mathbf{P}$ mon endocrine disorder after diabetes and hyperthyroidism (1). The prevalence in women over the age of $50 \mathrm{y}$ is about $2 \%$ (2). The condition is often diagnosed in asymptomatic subjects during a routine serum calcium measurement $(1,3)$. PHPT is classically associated with an elevated level of total serum calcium (after adjustment for albumin concentration) and of serum parathyroid hormone (PTH). However, in some patients PTH levels may be normal but inappropriate to hypercalcemia. Patients with vitamin D insufficiency may have higher PTH levels, and thus 25-hydroxyvitamin D should be measured. One important differential diagnosis is familial hypocalciuric hypercalcemia, because surgery is not warranted for this condition, which is due to heterozygous calcium-sensing receptor gene mutations and is associated with a low urinary calcium level.

The clinical signs and symptoms that are often associated with PHPT involve the skeleton (osteoporotic fractures, pain, bone lesions, chondrocalcinosis), the kidneys (nephrolithiasis, nephrocalcinosis, impaired renal function), and, more rarely now, the digestive tract (peptic ulcers, constipation, pancreatitis) and the cardiovascular system (hypertension) (1-4). PHPT may also cause neuropsychiatric symptoms, cognitive impairments, and decreased quality of life. Hypercalcemic crisis is rare but is a life-threatening medical emergency.

Patients with asymptomatic PHPT may remain stable over many years or show progression. After $15 \mathrm{y}$ of prospective followup, about a third of subjects develop additional signs of the disease (e.g., kidney stones, worsening hypercalcemia, reduced bone mineral density, or fragility fractures) (3).

Surgery is the only curative treatment for PHPT. According to recent recommendations (4), surgery is indicated in any patient 
younger than $50 \mathrm{y}$, any symptomatic patient, and asymptomatic patients with any of the following at diagnosis or during followup: a serum calcium level more than $0.25 \mathrm{mmol} / \mathrm{L}(>1 \mathrm{mg} / \mathrm{dL})$ above the upper limit of normal, a calculated creatinine clearance rate of less than $60 \mathrm{~mL} / \mathrm{min}$, a $24 \mathrm{~h}$-urine calcium level higher than $10 \mathrm{mmol}(>400 \mathrm{mg})$ and increased risk of stones, nephrolithiasis or nephrocalcinosis seen on imaging (radiography, ultrasound, or $\mathrm{CT}$ ), osteoporosis ( $\mathrm{T}$ score of -2.5 or less in the lumbar spine, total hip, femoral neck, or distal third of the radius, measured by dual-energy x-ray absorptiometry), or a vertebral fracture seen on imaging studies.

Although a single parathyroid adenoma is the most frequent occurrence, multiglandular disease (MGD) with two, sometimes three, adenomas or multiglandular hyperplasia is present in $15 \%-$ $20 \%$ of cases $(1,5)$. Parathyroid carcinoma is rare $(<1 \%$ of cases of PHPT) and is associated with a 10-y recurrence-free and diseasespecific survival of $69 \%$ and $91 \%$, respectively (6).

MGD can be either sporadic or hereditary, such as in isolated familial PHPT or in MEN1 syndrome (menin gene MEN1 mutation), MEN2A syndrome (RET oncogene mutation), MEN4 syndrome (CDKN1B mutation), and hyperparathyroidism-jaw tumor syndrome (HRPT2/CDC73 mutation; risk of parathyroid carcinoma) (7). MEN2B (also called MEN3) is rarely associated with PHPT. Parathyroid involvement is observed in 95\% of MEN1 cases and is most often the initial manifestation. A correct diagnosis of MEN1 is important for performing adequate initial surgery and for early detection of other associated endocrinopathies (pancreaticoduodenal, pituitary, or thymic tumors and occasional bronchial carcinoids) $(7,8)$. MEN1 must be suspected in cases of MGD or in cases of PHPT in subjects younger than $30 \mathrm{y}$. Longterm treatment with lithium or a history of neck radiation is also associated with MGD.

About $10 \%-15 \%$ of healthy subjects harbor a fifth parathyroid gland (9). Supernumerary glands can cause recurrent disease (e.g., in MEN1).

Conventional surgery for PHPT relies on inspection of the 4 parathyroid glands through bilateral cervical exploration (5). In recent years, there has been a significant shift toward targeted operations (10), which may be performed during open surgery $(7,10)$ or by endoscopic approaches $(11)$. This shift has been driven by two technologic developments: more sensitive imaging techniques, which enabled preoperative detection of the lesions, and intraoperative PTH monitoring, to verify the absence of other hyperactive glands (12).

Proposed by the team of O'Doherty and Coakley (13), ${ }^{99 m} \mathrm{Tc}-$ sestamibi scintigraphy rapidly gained widespread acceptance and rendered ${ }^{201} \mathrm{Tl}$ parathyroid scanning obsolete. It was recognized as the most sensitive noninvasive imaging study for PHPT (14-16). Parathyroid ultrasound is often used as a complementary imaging technique, although its effectiveness depends on the operator's skill (10). As stated in the parathyroid imaging guidelines of the European Association of Nuclear Medicine (EANM) and the Society of Nuclear Medicine and Molecular Imaging (SNMMI), imaging is optional before bilateral surgery for PHPT but is strongly recommended before targeted surgery or in cases of reintervention $(17,18)$. Surgery for hyperparathyroidism secondary to renal failure is not discussed here. In these patients, preoperative scintigraphy can help select the least autonomous parathyroid tissue for preservation and identify ectopic or supernumerary parathyroid glands (19-21), which are the main cause of recurrent disease (22).
This review article addresses some key points related to imaging of PHPT. The article is the product of collaboration between nuclear physicians, an endocrinologist, and endocrine surgeons and complies with the ethical guidelines of the authors' institutions.

\section{USEFULNESS OF PREOPERATIVE IMAGING BEFORE CONVENTIONAL BILATERAL SURGERY}

Without imaging, bilateral cervical exploration is curative in $92 \%-$ $95 \%$ of patients who undergo surgery for PHPT, as shown by older studies done before modern imaging was available (5). Indeed, an experienced surgeon can identify most pathologic glands (9,23-25).

However, preoperative imaging allows selection of patients eligible for targeted surgery and identification of ectopic glands. The incidence of ectopic glands is estimated at $6 \%-16 \%$ (24). Most ectopic glands present minor ectopia (e.g., in thyrothymic ligaments, in the tracheoesophageal groove, or partially embedded in the thyroid) and are easily located by an experienced surgeon. However, some parathyroid glands present major ectopia, which may lead to surgical failure, even for experienced surgeons, and can be a source of medical litigation. For example, $1 \%-2 \%$ of subjects have ectopic glands that require thoracic surgery instead of cervical surgery $(23,24)$.

In agreement with recommendations (16), we think that preoperative imaging should always be performed. Good imaging should be effective, provide morphologic and functional information, be noninvasive, and not be too expensive. These conditions can be fulfilled by combining cervical ultrasound and scintigraphy.

\section{Positive Parathyroid Scans}

Even in the case of classic bilateral cervicotomy, preoperative imaging has several benefits. It reduces the extent of anatomic dissection or unnecessary thyroid resections and morbidity, especially with regard to recurrent laryngeal nerve palsy, hypoparathyroidism, and hematomas. It reduces operation time; the surgeon starts by exploring the suspected site and the tumor can be sent for pathologic examination while the surgeon proceeds with inspection of the remaining parathyroid glands (26). It detects ectopic cervical glands without the need for extensive dissections. It detects thoracic glands that are not accessible through the neck (26).

In a retrospective series of 202 resected ectopic glands (major and minor ectopias), preoperative scintigraphy had a sensitivity of $89 \%$ and a positive predictive value of $90 \%$. Among 59 patients who underwent both scintigraphy and ultrasound, scintigraphy was superior to ultrasound in 28 patients and inferior in 3 (27). Ultrasound detection is hampered in cases of a retrotracheal, retroesophageal, or intrathoracic parathyroid gland.

Ectopias can be acquired or congenital. Tumors from superior parathyroid glands (also named P4) may prolapse along the tracheoesophageal groove into the lower neck or upper mediastinum and can be found in a para- or retroesophageal position. By contrast, ectopias of the lower parathyroids (P3) are often congenital and are due to an abnormal migration of the glands. They may be near the carotid and jugular vessels in the neck or inside the thymus in the anterior mediastinum. More rarely, pathologic glands can be associated with the vagal nerve within the carotid sheath or in the aortopulmonary window. Parathyroid glands completely buried in the thyroid parenchyma may be derived from P3 or P4 or may be supernumerary.

Suspecting a MGD on preoperative imaging may help the surgeon plan the surgical approach. 
Finally, although thyroid nodules may be a cause of falsepositives on parathyroid scintigraphy, it should not be forgotten that a ${ }^{99 \mathrm{~m}} \mathrm{Tc}$-sestamibi-positive, ${ }^{123}$ I-iodine-negative thyroid nodule carries a risk of malignancy (28) and may justify fine-needle biopsy before parathyroid surgery.

\section{Negative Parathyroid Scans}

In principle, imaging results should not influence the surgical decision (12,29). However, some recent studies (30-32), although not all (33), reported that surgical failure rate is higher when preoperative imaging findings are negative.

According to a multicenter study, patients with negative ultrasound and scintigraphy results had smaller parathyroid lesions and a higher proportion of hyperplasia (22\%). Moreover, cervicotomy involved a higher number of extended surgical procedures, such as thyroidectomy and thymectomy. Finally, PHPT was still present in $18 \%$ of subjects after surgery (30). Even in a center with great experience, the Mayo Clinic, the surgical failure rate in scintigraphy-negative patients can be as high as $10 \%$ despite the use of an intraoperative PTH assay (31).

Thus, negative imaging results are also informative and indicate that an experienced multidisciplinary team, including a surgeon, an endocrinologist, and imaging specialists, should be entrusted with the case.

\section{ADVANTAGES AND DISADVANTAGES OF TARGETED SURGERY}

Targeted parathyroidectomy has several advantages: a lower rate of complications, such as hypoparathyroidism, nerve injury, or unaesthetic scars; a shorter operation time; and a higher possibility of outpatient surgery and local anesthesia.

The option of targeted surgery should, however, be entertained only if the rate of failure (e.g., unrecognized MGD) is acceptably low. A Swedish randomized study (34) showed an advantage for unilateral surgery, in terms of operative time and incidence of transient hypocalcemia, whereas the rate of failure (persistent or recurrent hyperparathyroidism) was not significantly higher $(8.5 \%$ for unilateral surgery vs. $4.5 \%$ for bilateral surgery). However, a potential bias to these comparisons is that patients randomized for bilateral surgery did not undergo scintigraphy (26).

To reduce the risk of failure, several institutions associate targeted surgery with intraoperative PTH monitoring (7). PTH has a short halflife in plasma (2-4 min). According to the widely used Miami criterion (35), a 50\% decrease in PTH levels 10 min after removal of the putative lesion suggests a curative surgery. However, the procedure may sometimes yield inaccurate results, as when PTH concentrations decrease even in the presence of a second smaller lesion (36,37). The prevalence of MGD, estimated on the basis of intraoperative PTH monitoring, is about 5\% (35), much lower than the conventional rate of $15 \%-20 \%(5,38)$. Intraoperative PTH monitoring may also wrongly lead to bilateral surgery, because of delayed decay of PTH in cases of excessive mobilization of the adenoma before its removal or slow elimination kinetics in some patients.

Although recommended when performing targeted parathyroidectomy (7), rapid PTH assays are not available to most surgeons worldwide (7). Moreover, PTH monitoring prolongs operation time and limits the feasibility of local anesthesia. As an alternative to intraoperative PTH, some surgeons recommend inspection of the ipsilateral gland (39). In the case of double-adenomas, however, bilateral distribution is expected, not only because of prob- ability (two thirds of the cases) but also because they often originate from the two superior glands (40).

Another point to consider is that targeted surgery may require conversion to bilateral surgery in about $20 \%$ of cases $(41,42)$. Conversion is often due to imaging failure: wrong localization or MGD missed by imaging and identified by inspection of the ipsilateral gland or intraoperative PTH monitoring (41).

In summary, as stated in the SNMMI and EANM guidelines, a good preoperative imaging study can play a major role in the selection of candidates for targeted surgery $(17,18)$.

\section{PERFORMANCE OF DIFFERENT SCINTIGRAPHIC PROTOCOLS TO GUIDE TARGETED SURGERY}

A valuable presurgical imaging technique should be able to identify MGD, limit the rate of false-positives (e.g., thyroid nodules), and detect small lesions. Small parathyroid lesions are increasingly frequent because of earlier detection of PHPT. In the study of Almquist et al., the median weight of adenomas decreased from $750 \mathrm{mg}$ for women who underwent surgery between 1990 and 1995 to $520 \mathrm{mg}$ for those who underwent surgery between 2000 and 2007 (43).

Ultrasound is not accurate enough to be used alone for targeted surgery. It has a low sensitivity for MGD and ectopic lesions. In the study by Haber et al. (44), ultrasound recognized only $17 \%$ of MGD cases (1/6) and $25 \%$ of ectopic glands (2/8).

Although a metaanalysis showed that scintigraphy is superior to ultrasound, its sensitivity decreases in the case of MGD $(88.44 \%$ for single adenoma, $44.46 \%$ for hyperplasia, and $29.95 \%$ for dual adenoma). Moreover, sensitivity varies greatly among different studies (15). A description of the single-tracer dual-phase and dual-tracer subtraction parathyroid scintigraphy protocols can be found in the EANM and SNMMI guidelines $(17,18)$. The techniques are also summarized in Supplemental Table 1 (available at http://jnm.snmjournals.org).

Dual-phase ${ }^{99 \mathrm{~m}} \mathrm{Tc}$-sestamibi scintigraphy, generally using a parallel collimator and imaging at $15 \mathrm{~min}$ and 2-3 h, cannot detect MGD most of the time $(45,46)$. Indeed, many hyperplastic parathyroid lesions do not significantly retain the tracer at a late time. Among the 11 MGD patients of the study by Martin et al., doublephase scintigraphy wrongly suggested a single adenoma in 9 cases and was negative in the remaining 2 cases (46). In addition, this technique has a high rate of false-positives because of the absence of a thyroid-specific tracer. The sensitivity of ${ }^{99 \mathrm{~m} T c-s e s t a m i b i}$ single-tracer scintigraphy can be improved by adding pinhole acquisitions and SPECT/CT (47). The percentage of detected MGD cases remains, however, limited. Therefore, we think that single-tracer dual-phase scintigraphy cannot reliably guide targeted parathyroid surgery.

For the same reason, the use of intraoperative $\gamma$ probes to opt for targeted surgery has severe limits. Murphy and Norman argued that any excised tissue containing more than $20 \%$ of background activity, in a patient with positive ${ }^{99 \mathrm{~m}} \mathrm{Tc}$-sestamibi results, is likely to be a solitary adenoma (48). However, because MGD is rarely seen on late scintigraphic images $(17,46)$, this approach was recently abandoned by the very team who first proposed it (49).

\section{Importance of Simultaneous Dual-Tracer Imaging}

Hindié et al. proposed a ${ }^{99 \mathrm{~m}} \mathrm{Tc}-$ sestamibi/ ${ }^{123} \mathrm{I}$ subtraction protocol based on the simultaneous acquisition of the two tracers (instead of a sequential acquisition) (26). Dual-isotope acquisition 


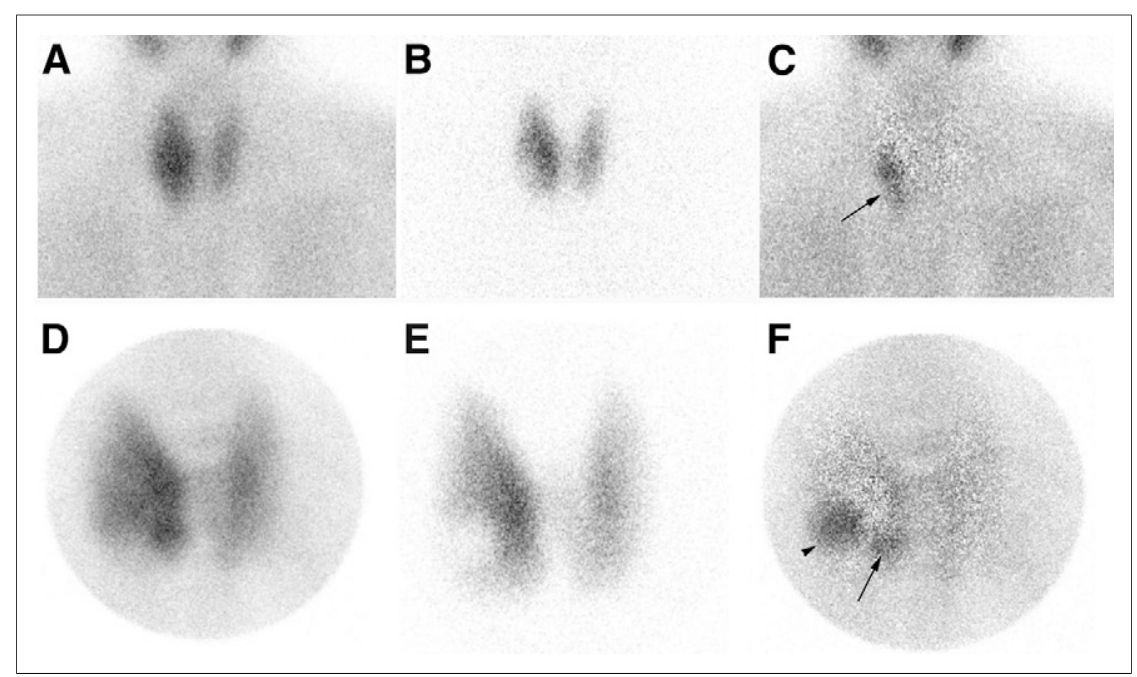

FIGURE 1. Simultaneous dual-tracer ${ }^{99 m T C-s e s t a m i b i}$ and ${ }^{123}$ scan in 55 -y-old patient with PHPT: parallel-hole collimator images with ${ }^{99 m T c}$-sestamibi (A), ${ }^{123}$ (B), and subtraction (C) and pinhole images of neck with ${ }^{99 \mathrm{mT}} \mathrm{Tc}-$ sestamibi $(\mathrm{D}),{ }^{123}(\mathrm{E})$, and subtraction (F). Subtraction on parallel-hole collimator images (C) shows residual focus on right side (arrow), which is difficult to interpret. Pinhole images offer better resolution. After subtraction (F), 2 foci are clearly seen on right side, one corresponding to ${ }^{123} \mid$-cold thyroid nodule (arrowhead) and the other, at inferior pole, corresponding to parathyroid lesion (arrow).

is possible using two narrow and nonoverlapping energy windows. This approach minimizes motion artifacts, increases sensitivity, and reduces acquisition time (50). In addition to the cervicomediastinal image, a magnified view of the thyroid bed with a pinhole collimator increases spatial resolution and thus sensitivity to detect hyperfunctioning parathyroid glands (Fig. 1) (50). The advantages of dualisotope subtraction scintigraphy have been highlighted by several recent studies (51-54). In a comparative analysis on 37 patients (41 lesions), Caveny et al. confirmed the superiority of the simultaneous dual-tracer technique over the single-tracer double-phase

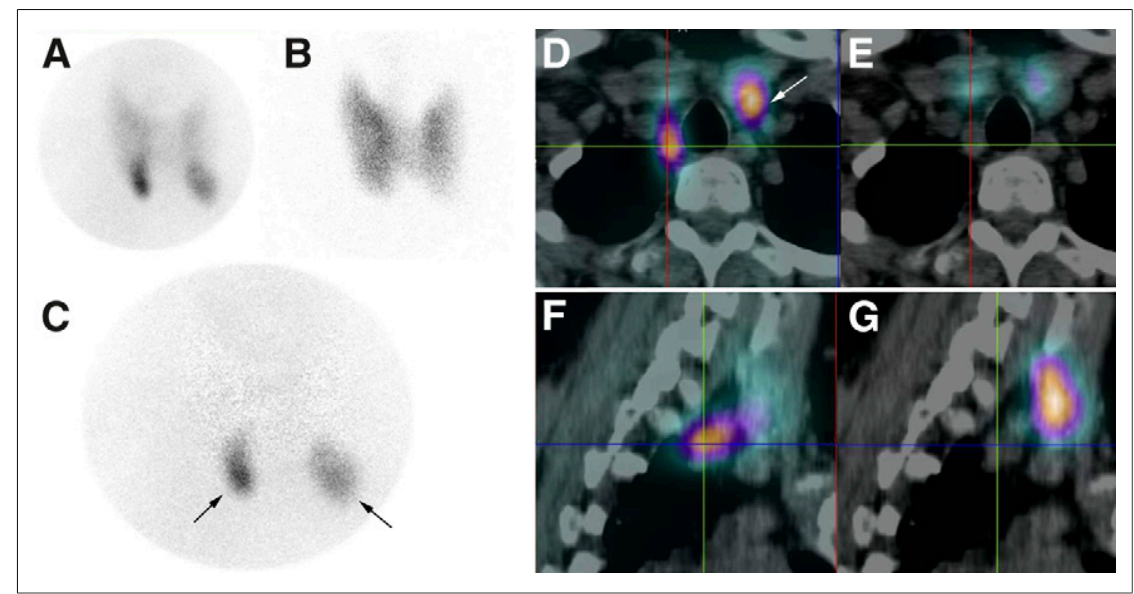

FIGURE 2. Simultaneous dual-tracer ${ }^{99 m T C-s e s t a m i b i ~ a n d ~}{ }^{123}$ s scan in $42-y$-old patient with PHPT. In planar pinhole images with ${ }^{99 \mathrm{mTC}} \mathrm{T}-\mathrm{sestamibi}(\mathrm{A}),{ }^{123}$ (B), and subtraction (C), subtraction image shows 2 residual foci in both inferior poles of thyroid (arrows). ${ }^{99 m T C-s e s t a m i b i ~ S P E C T / C T ~}$ images in axial (D) and sagittal $(F)$ views reveal that parathyroid lesion on left side corresponds to orthotopic inferior (P3) parathyroid gland (arrow), whereas lesion on right side (cross-marked) is located posteriorly, behind thyroid and trachea. This is a typical feature of a prolapsed superior (P4) parathyroid gland, which can be difficult to detect on ultrasound. Corresponding ${ }^{123}$ I SPECT/ $\mathrm{CT}$ images ( $\mathrm{E}$ and $\mathrm{G})$ confirm absence of ${ }^{123}$ |-iodine uptake in both parathyroid lesions. protocol in terms of both sensitivity (94\% vs. $66 \%, P<0.01)$ and number of falsepositive foci ( 0 vs. 2). The authors also suggested that, in light of the information obtained from subtraction images, the late ${ }^{99 \mathrm{~m}}$ Tc-sestamibi image did not provide any additional information (on the contrary, it reduced the sensitivity and confidence in the interpretation of the data) (51). The same team also underscored the importance of adding a pinhole acquisition; the use of a parallel collimator alone would result in a loss of about 20 points of sensitivity (53).

Simultaneous dual-isotope acquisition has high sensitivity for the identification of MGD (Fig. 2) (55). Careful image analysis is nevertheless required because MGD is commonly characterized by the presence of glands of unequal size and unequal intensity of tracer uptake (55). Thus, a pinhole view is necessary for optimal sensitivity $(17,53,54)$. Moreover, image subtraction should be carefully monitored. A subtraction by gradual steps is preferable to avoid having smaller glands disappear into the background (17). If not available, ${ }^{123} \mathrm{I}$ can be replaced by ${ }^{99 \mathrm{~m}} \mathrm{Tc}$-pertechnetate; its sensitivity for MGD detection is about $60 \%(56,57)$. In fact, the use of ${ }^{99 \mathrm{~m}} \mathrm{Tc}$-pertechnetate precludes the acquisition of a simultaneous image and the subtraction image may thus suffer from motion artifacts. Moreover, dual-isotope SPECT/CT acquisition would not be possible.

Dual-tracer imaging should be performed at least 3 wk after radiologic examinations involving administration of ${ }^{123}$ I-iodine contrast medium. Also, thyroid hormone replacement therapy should be withheld for 2 wk before imaging. This is necessary even in cases of previous thyroidectomy, as ${ }^{123}$ I would allow the correct identification of residual thyroid tissue, which can be mistaken for an enlarged parathyroid on ${ }^{99} \mathrm{~m}$ Tc-sestamibi single-tracer scintigraphy or neck ultrasound (Fig. 3).

\section{The Role of Dual-Isotope SPECT/CT}

Tomographic imaging (SPECT or SPECT/ CT) of the parathyroids is less sensitive than, and therefore cannot replace, pinhole imaging, especially because the shoulders are in the field of view (58). In a prospective study by Neumann et al., subtraction SPECT/CT had an overall sensitivity of $70 \%$ (59). Subtraction is more complex with dual-isotope tomographic images than with pinhole planar images. In our clinical practice, we prefer to visually compare the 3 tomographic projections of ${ }^{99} \mathrm{~m}$ Tc-sestamibi and ${ }^{123} \mathrm{I}$ images, without subtraction. We perform subtraction only on pinhole images (Fig. 2).

However, complementary SPECT/CT provides useful information $(59,60)$, such as the correct anatomic localization of a lesion 


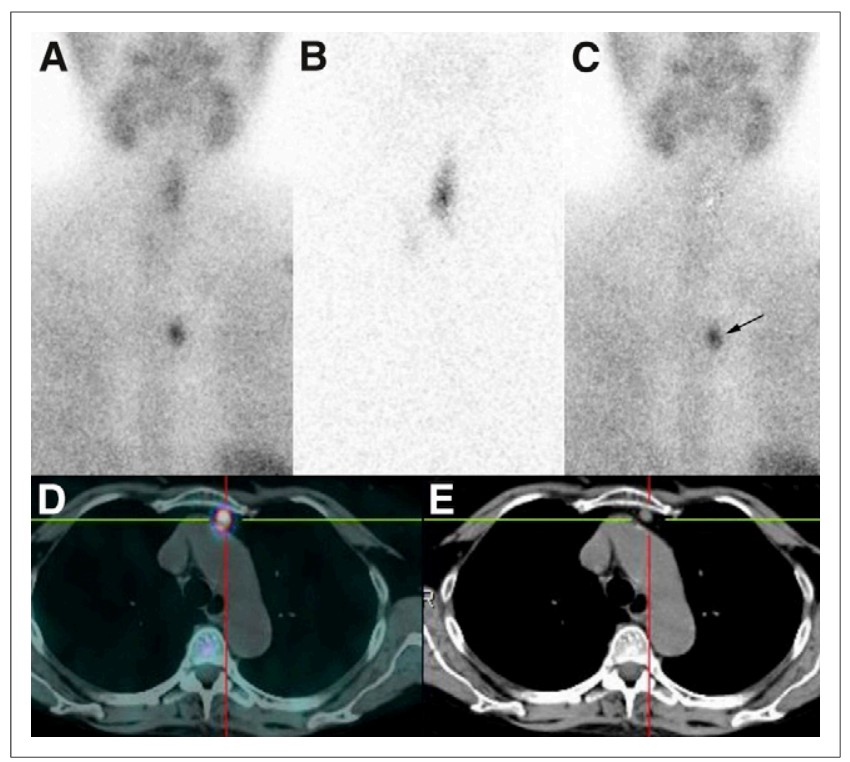

FIGURE 3. Patient with PHPT and previous history of subtotal thyroidectomy for Graves disease. Neck ultrasound suggested parathyroid lesion on lower right side. Simultaneous dual-tracer acquisition of ${ }^{99 \mathrm{mT}} \mathrm{Tc}$-sestamibi and ${ }^{123}$ images was performed after thyroid hormone replacement therapy had been withheld for 2 wk. In large-field-of-view parallel-hole collimator images of ${ }^{99 m T C}$-sestamibi (A), ${ }^{123 \mid}$ (B), and subtraction (C), suggestive lesion on ultrasound seems to correspond to thyroid remnant, with no visible neck foci on subtraction image (also confirmed in pinhole view). Intense focus of ${ }^{99 m} \mathrm{Tc}$-sestamibi uptake is seen in thorax. Axial ${ }^{99 m T c-}$ sestamibi SPECT/CT fusion image (D) and corresponding CT view (E) identify $9 \times 9 \times 15 \mathrm{~mm}$ mediastinal lesion in front of ascending aorta. At surgery, it corresponded to 537-mg parathyroid adenoma.

on the anteroposterior axis. For instance, posterior adenomas are preferably resected through a lateral approach. When SPECT/CT machines were not available, this information was less accurately obtained through anterior oblique or lateral pinhole views. Lateral views, and now SPECT/CT, can also help differentiate thyroid from parathyroid foci or identify a parathyroid lesion situated behind a thyroid nodule or behind another parathyroid lesion $(51,55,58)$.

Finally, SPECT/CT is useful for ectopic parathyroid lesions and to differentiate pathologic from physiologic foci of uptake (e.g., brown fat, muscle, or bone marrow).

\section{Multiphase 4-Dimensional (4D) CT Scanner}

Some reports suggest that 4D CT is superior to scintigraphy and recommend it as an initial localization study in PHPT (61). Despite its sophisticated images, there is still controversy over whether a 4D CT scanner can reliably identify MGD or differentiate thyroid nodules from parathyroid lesions, especially because, in the existing studies, 4D CT was not compared with subtraction scintigraphy. The sensitivity of 4D CT in diagnosing MGD was only $32 \%$ in the study by Kukar et al. (61) and $14 \%$ in the study by Madorin et al. (62). Because a 4D CT scanner uses a 3- or a 4-phase CT protocol, it delivers a high dose to the thyroid, which is problematic in younger patients (62) and requires injection of contrast medium in a population in which renal impairment is not uncommon.

\section{IMAGING STRATEGY BEFORE REOPERATION FOR PERSISTENT OR RECURRENT HYPERPARATHYROIDISM}

The decision to operate again should be made carefully, as the complication rate is higher than for a first surgery, even for experienced teams (63). Accurate preoperative localization is therefore essential.

First-line imaging should include both scintigraphy and neck ultrasound. Concordant localization by two modalities is required. In the case of a cervical image of uncertain origin, ultrasoundguided fine-needle aspiration for PTH assay may be performed (16). Contrast-enhanced CT (or MR imaging) is used to confirm a thoracic gland found by scintigraphy or if neck ultrasound and scintigraphy results are negative or inconclusive. A 4D CT scanner seems to offer good sensitivity, at the price of a high number of false-positives (e.g., exophytic thyroid nodules or lymph nodes) (64). When available, PET/CT with ${ }^{11} \mathrm{C}$-methionine, ${ }^{11} \mathrm{C}$-choline, or ${ }^{18} \mathrm{~F}$-fluorocholine can also be proposed. Finally, when imaging results are inconclusive, invasive procedures such as selective venous sampling with PTH measurements may be warranted (16), but the yield is highly dependent on the operator's expertise $(65,66)$. If the pathologic gland cannot be identified beforehand, the rationale for reoperation is questionable and medical treatment (e.g., with calcimimetics) should be discussed.

Thus, ${ }^{99 \mathrm{~m}}$ Tc-sestamibi scintigraphy plays a major role in surgical decision making. Unfortunately, several studies have reported a low sensitivity. In a series of 19 reoperated patients (18 glands resected in 17 patients and 2 patients with negative surgery), the sensitivity of ${ }^{99 \mathrm{~m}} \mathrm{Tc}$-sestamibi scintigraphy (double-phase planar imaging plus SPECT) was $33 \%(6 / 18)$ and the positive predictive value $67 \%(6 / 9)$. This translated into a low cure rate (11/19 patients) (67). Again, the sensitivity of dual-tracer scintigraphy in patients with persistent hyperparathyroidism seems to be quite a bit higher than that of single-tracer scintigraphy, as shown in a prospective study by Schalin-Jantti et al. comparing different techniques for the localization of parathyroid glands (68). Reoperation, after taking into account the results of the various techniques, cured 18 of 21 patients $(86 \%)$ and led to the resection of 19 pathologic glands. The sensitivity of subtraction scintigraphy (planar imaging with a parallel-hole collimator only) was 59\% and that of single-tracer ${ }^{99 \mathrm{~m} T c-s e s t a m i b i}$ (double-phase plus SPECT/CT) was $19 \%(P<0.01)$. There were no false-positives with either technique. Selective venous sampling with PTH assay yielded a sensitivity of only $40 \%$ with 9 false-positives, including the 3 patients in whom reoperation was unfruitful (68).

\section{PARATHYROID IMAGING WITH NOVEL PET TRACERS}

${ }^{11} \mathrm{C}$-methionine seems to be a sensitive tracer for imaging patients before reoperation $(65,68,69)$. In a study by Schalin-Jantti et al., the sensitivity of ${ }^{11} \mathrm{C}$-methionine PET was comparable to that of subtraction scintigraphy (65\% with one false-positive vs. $59 \%$ with no false-positive) (68). In another series that investigated patients with previous neck surgery and negative singletracer ${ }^{99 \mathrm{~m} T c}$-sestamibi SPECT/CT results, ${ }^{11} \mathrm{C}$-methionine PET/ CT was positive in 6 of 15 cases (40\%) (69). However, because of restricted availability and cost, there probably is little role for routine use of ${ }^{11} \mathrm{C}$-methionine in PHPT patients undergoing a first operation. Moreover, preliminary data suggest that the sensitivity of ${ }^{11} \mathrm{C}$-methionine in MGD patients is limited (70).

${ }^{18} \mathrm{~F}$-FDG PET has poor sensitivity in unselected PHPT patients. However, it can offer complementary information to ${ }^{99 \mathrm{~m}} \mathrm{Tc}$-sestamibi in patients with parathyroid carcinoma.

Recently, several short series of patients imaged with choline tracers have been published. Orevi et al. compared ${ }^{11} \mathrm{C}$-choline with ${ }^{99 \mathrm{~m}} \mathrm{Tc}$-sestamibi/ $/ 99 \mathrm{~m} \mathrm{Tc}$-pertechnetate scintigraphy in 40 patients 


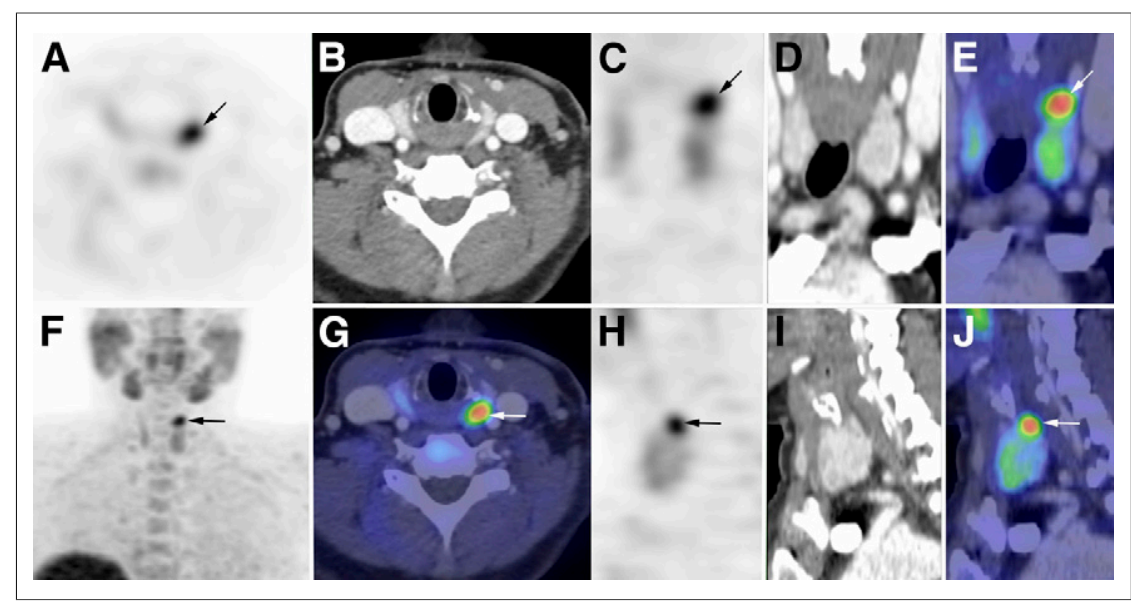

FIGURE 4. ${ }^{18} \mathrm{~F}$-fluorocholine PET/CT (contrast-enhanced CT) performed $20 \mathrm{~min}$ after intravenous injection of ${ }^{18} \mathrm{~F}$-fluorocholine ( $3 \mathrm{MBq} / \mathrm{kg}$ ) in patient with PHPT and doubtful results on ${ }^{99 \mathrm{mTC}} \mathrm{T}$ sestamibi scintigraphy performed at another institution (not repeated). PET, CT, and PET/CT images are displayed in axial (A, B, and G), coronal (C, D, and E), and sagittal $(\mathrm{H}, \mathrm{I}$, and J) views, as well as PET maximum-intensity projection (F). Choline-avid hyperfunctioning parathyroid gland is seen at upper pole of left thyroid lobe (arrow). Neck ultrasound confirmed presence of hypoechoic $10 \times 5 \times 12 \mathrm{~mm}$ nodule behind left upper pole of thyroid.

with hyperparathyroidism, 27 of whom underwent surgery. In 23 patients, both techniques correctly identified parathyroid lesions. Of the 4 discordant cases, one was a ${ }^{99 m} \mathrm{Tc}$-sestamibi false-positive and an ${ }^{11} \mathrm{C}$-choline true-positive and two were negative on ${ }^{99 \mathrm{~m}} \mathrm{Tc}$ sestamibi with false-positive findings on ${ }^{11} \mathrm{C}$-choline $(71)$.

In view of the wider availability of ${ }^{18} \mathrm{~F}$-fluorocholine, recent findings with this tracer are of particular interest. Lezaic et al. compared ${ }^{18} \mathrm{~F}$-fluorocholine (with imaging at 5 and $60 \mathrm{~min}$ ) to ${ }^{99 \mathrm{~m} T c-s e s t a m i b i}$ (double-phase imaging plus early SPECT/CT and ${ }^{99 \mathrm{~m}} \mathrm{Tc}$-pertechnetate thyroid scanning). In 24 patients undergoing surgery for PHPT, surgery identified 39 lesions (17 patients had a solitary adenoma and 7 had MGD). ${ }^{18} \mathrm{~F}$-fluorocholine images were better defined at $60 \mathrm{~min}$ than at $5 \mathrm{~min}$ (better lesionto-background and lesion-to-thyroid contrast). ${ }^{18} \mathrm{~F}$-fluorocholine showed higher sensitivity than ${ }^{99 \mathrm{~m}} \mathrm{Tc}$-sestamibi scintigraphy (92\% vs. 64\%) (72). Although the findings from this study are appealing, the sensitivity of ${ }^{99 \mathrm{~m}} \mathrm{Tc}$-sestamibi was lower than would be expected in previously unoperated PHPT patients (51). In another series, ${ }^{18} \mathrm{~F}$-fluorocholine with both dynamic and static acquisitions was used in patients with discordant findings at ultrasonography and subtraction scintigraphy. In the 8 patients with PHPT who underwent surgery, ${ }^{18} \mathrm{~F}$-fluorocholine offered good sensitivity, although its results mostly confirmed the findings of ${ }^{99 \mathrm{~m} T c-s e s t a m i-}$ bi/123I subtraction imaging (Table 2 of Michaud et al. (73)). Parathyroid lesions were more clearly seen on ${ }^{18} \mathrm{~F}$-fluorocholine in 2 patients and on ${ }^{99 \mathrm{~m}} \mathrm{Tc}$-sestamibi//23I subtraction in 1 patient. In 1 patient, ${ }^{18} \mathrm{~F}$-fluorocholine identified a contralateral lesion not seen on ${ }^{99 \mathrm{~m}} \mathrm{Tc}$-sestamibi/123I subtraction, which turned out, however, to be a false-positive.

Larger prospective studies are needed to compare ${ }^{18} \mathrm{~F}$-fluorocholine PET/CT with state-of-the-art conventional scintigraphy to clarify the role of choline imaging in specific settings (e.g., reoperative PHPT or patients undergoing their first operation with inconclusive imaging).

A potential advantage of ${ }^{18} \mathrm{~F}$-fluorocholine imaging is the higher resolution associated with PET technology (Fig. 4) and the shorter imaging time. Potential drawbacks are the higher cost and the absence of a specific thyroid tracer, with risk of false-positive results from thyroid nodules and inflammatory lymph nodes. Administration of intravenous contrast medium during choline PET/CT imaging may further improve results, especially in cases of recurrent disease.

\section{CONCLUSION}

The long-term cure rate of parathyroid surgery depends on the ability to resect hyperfunctioning parathyroid tumors and to identify MGD and ectopic glands. Preoperative detection of pathologic glands would avoid inappropriate surgery and reduce the rate of surgical conversion or failure. Subtraction scintigraphy after simultaneous acquisition of ${ }^{99} \mathrm{~m}$ Tc-sestamibi and ${ }^{123}$ I images provides better sensitivity than double-phase ${ }^{99 \mathrm{~m}} \mathrm{Tc}$-sestamibi scanning. Imaging with a pinhole collimator optimizes resolution. Complementary SPECT/CT images are of great value for anatomic localization and should be obtained routinely before reoperation to identify ectopic glands. Choline PET tracers may open new venues for radionuclide imaging of parathyroid glands.

\section{REFERENCES}

1. al Zahrani A, Levine MA. Primary hyperparathyroidism. Lancet. 1997;349: 1233-1238.

2. Lundgren E, Rastad J, Thrufjell E, Akerstrom G, Ljunghall S. Populationbased screening for primary hyperparathyroidism with serum calcium and parathyroid hormone values in menopausal women. Surgery. 1997;121:287294.

3. Rubin MR, Bilezikian JP, McMahon DJ, et al. The natural history of primary hyperparathyroidism with or without parathyroid surgery after 15 years. J Clin Endocrinol Metab. 2008;93:3462-3470.

4. Bilezikian JP, Brandi ML, Eastell R, et al. Guidelines for the management of asymptomatic primary hyperparathyroidism: summary statement from the fourth international workshop. J Clin Endocrinol Metab. 2014;99:35613569.

5. Russell CF, Edis AJ. Surgery for primary hyperparathyroidism: experience with 500 consecutive cases and evaluation of the role of surgery in the asymptomatic patient. Br J Surg. 1982;69:244-247.

6. Villar-del-Moral J, Jimenez-Garcia A, Salvador-Egea P, et al. Prognostic factors and staging systems in parathyroid cancer: a multicenter cohort study. Surgery. 2014; 156:1132-1144.

7. Udelsman R, Akerstrom G, Biagini C, et al. The surgical management of asymptomatic primary hyperparathyroidism: proceedings of the fourth international workshop. J Clin Endocrinol Metab. 2014;99:3595-3606.

8. Goudet P, Murat A, Binquet C, et al. Risk factors and causes of death in MEN1 disease: a GTE (Groupe d'Etude des Tumeurs Endocrines) cohort study among 758 patients. World J Surg. 2010;34:249-255.

9. Akerström G, Malmaeus J, Bergstrom R. Surgical anatomy of human parathyroid glands. Surgery. 1984;95:14-21.

10. Greene AB, Butler RS, McIntyre S, et al. National trends in parathyroid surgery from 1998 to 2008: a decade of change. J Am Coll Surg. 2009;209: $332-343$.

11. Henry JF, Sebag F, Cherenko M, Ippolito G, Taieb D, Vaillant J. Endoscopic parathyroidectomy: why and when? World J Surg. 2008;32:25092515.

12. Fraker DL, Harsono H, Lewis R. Minimally invasive parathyroidectomy: benefits and requirements of localization, diagnosis, and intraoperative PTH monitoringlong-term results. World J Surg. 2009;33:2256-2265. 
13. O'Doherty MJ, Kettle AG, Wells P, Collins RE, Coakley AJ. Parathyroid imaging with technetium-99m-sestamibi: preoperative localization and tissue uptake studies. J Nucl Med. 1992;33:313-318.

14. Hindié E, Melliere D, Simon D, Perlemuter L, Galle P. Primary hyperparathyroidism: is technetium $99 \mathrm{~m}$-sestamibi/iodine-123 subtraction scanning the best procedure to locate enlarged glands before surgery? J Clin Endocrinol Metab. 1995;80:302-307.

15. Ruda JM, Hollenbeak CS, Stack BC Jr. A systematic review of the diagnosis and treatment of primary hyperparathyroidism from 1995 to 2003. Otolaryngol Head Neck Surg. 2005;132:359-372.

16. Bergenfelz AO, Hellman P, Harrison B, Sitges-Serra A, Dralle H. Positional statement of the European Society of Endocrine Surgeons (ESES) on modern techniques in pHPT surgery. Langenbecks Arch Surg. 2009;394:761-764.

17. Hindié E, Ugur O, Fuster D, et al. 2009 EANM parathyroid guidelines. Eur J Nucl Med Mol Imaging. 2009;36:1201-1216.

18. Greenspan BS, Dillehay G, Intenzo C, et al. SNM practice guideline for parathyroid scintigraphy 4.0. J Nucl Med Technol. 2012;40:111-118.

19. Hindié E, Urena $P$, Jeanguillaume $C$, et al. Preoperative imaging of parathyroid glands with technetium-99m-labelled sestamibi and iodine-123 subtraction scanning in secondary hyperparathyroidism. Lancet. 1999;353:2200-2204.

20. Fuster D, Ybarra J, Ortin J, et al. Role of pre-operative imaging using ${ }^{99 \mathrm{~m}} \mathrm{Tc}$ MIBI and neck ultrasound in patients with secondary hyperparathyroidism who are candidates for subtotal parathyroidectomy. Eur J Nucl Med Mol Imaging. 2006;33:467-473.

21. Vulpio C, Bossola M, De Gaetano A, et al. Usefulness of the combination of ultrasonography and ${ }^{99 \mathrm{~m}} \mathrm{Tc}$-sestamibi scintigraphy in the preoperative evaluation of uremic secondary hyperparathyroidism. Head Neck. 2010;32: 1226-1235.

22. Hindié E, Zanotti-Fregonara P, Just PA, et al. Parathyroid scintigraphy findings in chronic kidney disease patients with recurrent hyperparathyroidism. Eur J Nucl Med Mol Imaging. 2010;37:623-634.

23. Levin KE, Clark OH. The reasons for failure in parathyroid operations. Arch Surg. 1989;124:911-914.

24. Phitayakorn R, McHenry CR. Incidence and location of ectopic abnormal parathyroid glands. Am J Surg. 2006;191:418-423.

25. Henry JF. Reoperation for primary hyperparathyroidism: tips and tricks. Langenbecks Arch Surg. 2010;395:103-109.

26. Hindié E, Melliere D, Perlemuter L, Jeanguillaume C, Galle P. Primary hyperparathyroidism: higher success rate of first surgery after preoperative Tc- $99 \mathrm{~m}$ sestamibi-I-123 subtraction scanning. Radiology. 1997;204:221-228.

27. Roy M, Mazeh H, Chen H, Sippel RS. Incidence and localization of ectopic parathyroid adenomas in previously unexplored patients. World J Surg. 2013;37: $102-106$.

28. Onkendi EO, Richards ML, Thompson GB, Farley DR, Peller PJ, Grant CS. Thyroid cancer detection with dual-isotope parathyroid scintigraphy in primary hyperparathyroidism. Ann Surg Oncol. 2012;19:1446-1452.

29. Mihai R, Simon D, Hellman P. Imaging for primary hyperparathyroidism: an evidence-based analysis. Langenbecks Arch Surg. 2009;394:765-784.

30. Bergenfelz AO, Wallin G, Jansson S, et al. Results of surgery for sporadic primary hyperparathyroidism in patients with preoperatively negative sestamibi scintigraphy and ultrasound. Langenbecks Arch Surg. 2011;396:83-90.

31. Dy BM, Richards ML, Vazquez BJ, Thompson GB, Farley DR, Grant CS. Primary hyperparathyroidism and negative Tc99 sestamibi imaging: to operate or not? Ann Surg Oncol. 2012;19:2272-2278.

32. Bagul A, Patel HP, Chadwick D, Harrison BJ, Balasubramanian SP. Primary hyperparathyroidism: an analysis of failure of parathyroidectomy. World J Surg. 2014;38:534-541.

33. Wachtel H, Bartlett EK, Kelz RR, Cerullo I, Karakousis GC, Fraker DL. Primary hyperparathyroidism with negative imaging: a significant clinical problem. Ann Surg. 2014;260:474-480.

34. Westerdahl J, Bergenfelz A. Unilateral versus bilateral neck exploration for primary hyperparathyroidism: five-year follow-up of a randomized controlled trial. Ann Surg. 2007;246:976-980.

35. Molinari AS, Irvin GL III, Deriso GT, Bott L. Incidence of multiglandular disease in primary hyperparathyroidism determined by parathyroid hormone secretion. Surgery. 1996;120:934-936.

36. Gauger PG, Agarwal G, England BG, et al. Intraoperative parathyroid hormone monitoring fails to detect double parathyroid adenomas: a 2-institution experience. Surgery. 2001;130:1005-1010.

37. Weber CJ, Ritchie JC. Retrospective analysis of sequential changes in serum intact parathyroid hormone levels during conventional parathyroid exploration. Surgery. 1999;126:1139-1143.

38. Lee NC, Norton JA. Multiple-gland disease in primary hyperparathyroidism: a function of operative approach? Arch Surg. 2002;137:896-899.
39. Cho NL, Gawande AA, Sheu EG, Moore FD Jr, Ruan DT. Critical role of identification of the second gland during unilateral parathyroid surgery: a prospective review of 119 patients with concordant localization. Arch Surg. 2011; 146:512-516

40. Milas M, Wagner K, Easley KA, Siperstein A, Weber CJ. Double adenomas revisited: nonuniform distribution favors enlarged superior parathyroids (fourth pouch disease). Surgery. 2003;134:995-1003.

41. Hessman O, Westerdahl J, Al-Suliman N, Christiansen P, Hellman P, Bergenfelz A. Randomized clinical trial comparing open with videoassisted minimally invasive parathyroid surgery for primary hyperparathyroidism. Br J Surg. 2010;97:177-184.

42. Hughes DT, Miller BS, Park PB, Cohen MS, Doherty GM, Gauger PG. Factors in conversion from minimally invasive parathyroidectomy to bilateral parathyroid exploration for primary hyperparathyroidism. Surgery. 2013; 154:1428-1434.

43. Almquist M, Bergenfelz A, Martensson H, Thier M, Nordenstrom E. Changing biochemical presentation of primary hyperparathyroidism. Langenbecks Arch Surg. 2010;395:925-928.

44. Haber RS, Kim CK, Inabnet WB. Ultrasonography for preoperative localization of enlarged parathyroid glands in primary hyperparathyroidism: comparison with ${ }^{99 \mathrm{~m}}$ technetium sestamibi scintigraphy. Clin Endocrinol (Oxf). 2002;57:241-249.

45. Taillefer R, Boucher Y, Potvin C, Lambert R. Detection and localization of parathyroid adenomas in patients with hyperparathyroidism using a single radionuclide imaging procedure with technetium-99m-sestamibi (double-phase study). J Nucl Med. 1992;33:1801-1807.

46. Martin D, Rosen IB, Ichise M. Evaluation of single isotope technetium 99Msestamibi in localization efficiency for hyperparathyroidism. Am J Surg. 1996; 172:633-636.

47. Ciappuccini R, Morera J, Pascal P, et al. Dual-phase ${ }^{99 \mathrm{~m}} \mathrm{Tc}$ sestamibi scintigraphy with neck and thorax SPECT/CT in primary hyperparathyroidism: a singleinstitution experience. Clin Nucl Med. 2012;37:223-228.

48. Murphy C, Norman J. The $20 \%$ rule: a simple, instantaneous radioactivity measurement defines cure and allows elimination of frozen sections and hormone assays during parathyroidectomy. Surgery. 1999;126:1023-1028.

49. Norman J, Lopez J, Politz D. Abandoning unilateral parathyroidectomy: why we reversed our position after 15,000 parathyroid operations. J Am Coll Surg. 2012;214:260-269.

50. Hindié E, Melliere D, Jeanguillaume C, Perlemuter L, Chehade F, Galle P. Parathyroid imaging using simultaneous double-window recording of technetium99m-sestamibi and iodine-123. J Nucl Med. 1998;39:1100-1105.

51. Caveny SA, Klingensmith WC III, Martin WE, et al. Parathyroid imaging: the importance of dual-radiopharmaceutical simultaneous acquisition with ${ }^{99 \mathrm{~m}} \mathrm{Tc}$ sestamibi and ${ }^{123}$ I. J Nucl Med Technol. 2012;40:104-110.

52. Tunninen V, Varjo P, Schildt J, et al. Comparison of five parathyroid scintigraphic protocols. Int J Mol Imaging. 2013;2013:921260.

53. Klingensmith WC III, Koo PJ, Summerlin A, et al. Parathyroid imaging: the importance of pinhole collimation with both single- and dual-tracer acquisition. $J$ Nucl Med Technol. 2013;41:99-104.

54. Guerin C, Lowery A, Gabriel S, et al. Preoperative imaging for focused parathyroidectomy: making a good strategy even better. Eur $J$ Endocrinol. 2015;172:519-526.

55. Hindié E, Melliere D, Jeanguillaume C, Urena P, deLabriolle-Vaylet C, Perlemuter L. Unilateral surgery for primary hyperparathyroidism on the basis of technetium Tc 99m sestamibi and iodine 123 subtraction scanning. Arch Surg. 2000;135:1461-1468.

56. Casara D, Rubello D, Pelizzo MR, Shapiro B. Clinical role of ${ }^{99} \mathrm{~m}^{\mathrm{TcO}}{ }_{4} / \mathrm{MIBI}$ scan, ultrasound and intra-operative gamma probe in the performance of unilateral and minimally invasive surgery in primary hyperparathyroidism. Eur J Nucl Med. 2001;28:1351-1359.

57. Nichols KJ, Tomas MB, Tronco GG, Palestro CJ. Sestamibi parathyroid scintigraphy in multigland disease. Nucl Med Commun. 2012;33:43-50.

58. Ho Shon IA, Yan W, Roach PJ, et al. Comparison of pinhole and SPECT ${ }^{99 \mathrm{~m}} \mathrm{Tc}-$ MIBI imaging in primary hyperparathyroidism. Nucl Med Commun. 2008;29: 949-955.

59. Neumann DR, Obuchowski NA, Difilippo FP. Preoperative ${ }^{123} \mathrm{I} /{ }^{99 \mathrm{~m}} \mathrm{Tc}$-sestamibi subtraction SPECT and SPECT/CT in primary hyperparathyroidism. J Nucl Med. 2008;49:2012-2017.

60. Delbeke D, Coleman RE, Guiberteau MJ, et al. Procedure guideline for SPECT/ CT imaging 1.0. J Nucl Med. 2006;47:1227-1234.

61. Kukar M, Platz TA, Schaffner TJ, et al. The use of modified four-dimensional computed tomography in patients with primary hyperparathyroidism: an argument for the abandonment of routine sestamibi single-positron emission computed tomography (SPECT). Ann Surg Oncol. 2015;22:139-145. 
62. Madorin CA, Owen R, Coakley B, et al. Comparison of radiation exposure and cost between dynamic computed tomography and sestamibi scintigraphy for preoperative localization of parathyroid lesions. JAMA Surg. 2013;148: 500-503.

63. Karakas E, Muller HH, Schlosshauer T, Rothmund M, Bartsch DK. Reoperations for primary hyperparathyroidism: improvement of outcome over two decades. Langenbecks Arch Surg. 2013;398:99-106.

64. Kelly HR, Hamberg LM, Hunter GJ. 4D-CT for preoperative localization of abnormal parathyroid glands in patients with hyperparathyroidism: accuracy and ability to stratify patients by unilateral versus bilateral disease in surgerynaive and re-exploration patients. AJNR. 2014;35:176-181.

65. Hessman O, Stalberg P, Sundin A, et al. High success rate of parathyroid reoperation may be achieved with improved localization diagnosis. World J Surg. 2008;32:774-781.

66. Ginsburg M, Christoforidis GA, Zivin SP, et al. Adenoma localization for recurrent or persistent primary hyperparathyroidism using dynamic four-dimensional CT and venous sampling. J Vasc Interv Radiol. 2015;26:79-86.

67. Witteveen JE, Kievit J, Stokkel MP, Morreau H, Romijn JA, Hamdy NA. Limitations of Tc99m-MIBI-SPECT imaging scans in persistent primary hyperparathyroidism. World J Surg. 2011;35:128-139.
68. Schalin-Jäntti C, Ryhanen E, Heiskanen I, et al. Planar scintigraphy with ${ }^{123} \mathrm{I} /{ }^{99 \mathrm{~m}} \mathrm{Tc}$-sestamibi, ${ }^{99 \mathrm{~m}} \mathrm{Tc}$-sestamibi SPECT/CT, ${ }^{11} \mathrm{C}$-methionine PET/CT, or selective venous sampling before reoperation of primary hyperparathyroidism? $J$ Nucl Med. 2013;54:739-747.

69. Traub-Weidinger T, Mayerhoefer ME, Koperek O, et al. ${ }^{11} \mathrm{C}$-methionine PET/CT imaging of ${ }^{99 \mathrm{~m}} \mathrm{Tc}-\mathrm{MIBI}-\mathrm{SPECT} / \mathrm{CT}$-negative patients with primary hyperparathyroidism and previous neck surgery. J Clin Endocrinol Metab. 2014;99:4199-4205.

70. Hayakawa N, Nakamoto Y, Kurihara K, et al. A comparison between ${ }^{11} \mathrm{C}$-methionine PET/CT and MIBI SPECT/CT for localization of parathyroid adenomas/hyperplasia. Nucl Med Commun. 2015;36:53-59.

71. Orevi M, Freedman N, Mishani E, Bocher M, Jacobson O, Krausz Y. Localization of parathyroid adenoma by ${ }^{11} \mathrm{C}$-choline PET/CT: preliminary results. Clin Nucl Med. 2014;39:1033-1038.

72. Lezaic L, Rep S, Sever MJ, Kocjan T, Hocevar M, Fettich J. ${ }^{18} \mathrm{~F}$-fluorocholine PET/ CT for localization of hyperfunctioning parathyroid tissue in primary hyperparathyroidism: a pilot study. Eur J Nucl Med Mol Imaging. 2014;41:2083-2089.

73. Michaud L, Burgess A, Huchet V, et al. Is ${ }^{18} \mathrm{~F}$-fluorocholine-positron emission tomography/computerized tomography a new imaging tool for detecting hyperfunctioning parathyroid glands in primary or secondary hyperparathyroidism? $J$ Clin Endocrinol Metab. 2014;99:4531-4536. 